\title{
FOTOQUÍMICA DE CHALCONAS FLUORADAS NO ESTADO SÓLIDO
}

\author{
Dari Cesarin-Sobrinho e José Carlos Netto-Ferreira* \\ Departamento de Química, Universidade Federal Rural do Rio de Janeiro, 23851-970, Seropédica - RJ
}

Recebido em 14/3/01; aceito em 20/6/01

\begin{abstract}
PHOTOCHEMISTRY OF FLUORINATED CHALCONES IN THE SOLID STATE. Chalcone and its fluorinated derivatives were synthesized and photolyzed in the solid state. UV irradiation of chalcone and its monosubstituted fluorine derivatives (3- and 4fluorchalcone) resulted in a mixture of anti-head-head ( $\gamma$-truxinic), sin-head-tail ( $\alpha$-truxilic) and anti-head-tail $(\varepsilon$-truxilic) dimers. On the other hand, upon irradiation of 3,4- and 3,5-difluorchalcone a stereoselective formation of the $\alpha$-truxilic photodimer was observed, whereas for 2-substituted chalcones (2,3-difluorchalcone, 2,5-difluorchalcone, 2,6-difluorchalcone and 2,3,4trifluorchalcone) the $\beta$-truxilic dimer was stereoselectively obtained. 2',3',4',5',6'-pentafluorchalcone was the less reactive of all chalcones studied and at least one of the possible photodimers, i.e the anti-head-head isomer, was identified. Irradiation of polyfluorinated chalcones such as 2,3,5,6-tetrafluor-, 2,3,4,5,6-pentafluor-, and 2,2',3,3',4,4',5,5',6,6'-decafluorchalcone led only to polymerization and/or decomposition products.
\end{abstract}

Keywords: chalcone; photodimerization; solid state.

\section{INTRODUÇÃO}

As fotorreações no estado cristalino têm sido estudadas desde o final do século XIX e vários trabalhos de revisão podem ser encontrados sobre o assunto ${ }^{1,2}$. Os trabalhos pioneiros nessa área tiveram início com Liebermann, em 1889, que estudou a dimerização de olefinas no estado cristalino ${ }^{3,4}$. Durante a primeira parte do século $\mathrm{XX}$, vários pesquisadores iniciaram a sistematização dos resultados da dimerização no estado cristalino a partir do estudo da fotoquímica de ácido cinâmico e derivados. Somente após os anos 60, com o aparecimento de técnicas de difração de raios-X, foi possível a Schmidt e colaboradores avançarem no entendimento dos processos de fotodimerização no estado cristalino, para os quais foram estabelcidas regras importantes, denominadas de regras topológicas ${ }^{5-8}$. De acordo com os princípios topoquímicos, as reações no estado sólido se processam com um mínimo de movimento atômico, o que implica que só serão esperadas reações de dimerização em compostos apresentando ligações olefínicas se as ligações duplas estiverem paralelas e orientadas de forma correta sob o ponto de vista estereoquímico.

Algumas reações no estado sólido aparentemente não seguem as regras topológicas e procedem ou através de defeitos ou em superfícies ou sítios onde o empacotamento cristalino é desordenado ${ }^{9-11}$.

Mais recentemente, e visando um melhor entendimento das fotorreações no estado cristalino, foi introduzido o conceito de cavidade reacional ${ }^{12}$, que representa qualitativamente o espaço ocupado pelo reagente. Assim, os movimentos atômicos que se seguem durante uma determinada reação exercem pressão sobre as paredes da cavidade, a qual torna-se distorcida, limitando tanto os movimentos de deslocamento molecular quanto as mudanças conformacionais que são permitidas durante a reação ${ }^{12}$. A nível molecular, tem sido mostrado que as reações no estado sólido somente podem ocorrer quando existe uma forte similaridade entre a estrutura dos reagentes, o estado de transição e o produto final ${ }^{12-14}$.
A fotólise de sistemas $\alpha, \beta$-insaturados que no estado cristalino apresentam distâncias intermoleculares de cerca de 4 A é capaz de levar à formação de ciclobutanos, como observado para ácido cinâmico e derivados ${ }^{15}$, cumarinas $^{16}$, chalconas e outras cetonas $\alpha, \beta$ insaturadas ${ }^{17,18}$.

A substituição por átomos de cloro, especialmente por dois átomos de cloro, em moléculas aromáticas tende a direcioná-las, quando no estado cristalino, a distâncias entre as ligações duplas de aproximadamente $4 \AA^{4}$, permitindo assim a formação de fotodímeros ${ }^{10}$. O mesmo tipo de comportamento foi observado quando da substituição de hidrogênios aromáticos por bromo ${ }^{19}$.

Esse trabalho tem como objetivo verificar a influência produzida pela substituição dos átomos de hidrogênio aromáticos por átomos de flúor em ambos os anéis, A e B, sobre a fotodimerização de chalconas no estado cristalino.

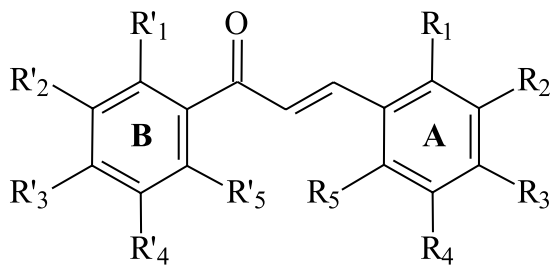

1: $\mathrm{R}_{1}=\mathrm{R}_{2}=\mathrm{R}_{3}=\mathrm{R}_{4}=\mathrm{R}_{5}=\mathrm{R}_{1}^{\prime}=\mathrm{R}_{2}^{\prime}=\mathrm{R}^{\prime}{ }_{3}=\mathrm{R}_{4}^{\prime}=\mathrm{R}^{\prime}{ }_{5}=\mathrm{H}$

2: $\mathrm{R}_{1}=\mathrm{R}_{3}=\mathrm{R}_{4}=\mathrm{R}_{5}=\mathrm{R}^{\prime}{ }_{1}=\mathrm{R}_{2}^{\prime}=\mathrm{R}^{\prime}{ }_{3}=\mathrm{R}_{4}^{\prime}=\mathrm{R}^{\prime}{ }_{5}=\mathrm{H} ; \mathrm{R}_{2}=\mathrm{F}$

3: $\mathrm{R}_{1}=\mathrm{R}_{2}=\mathrm{R}_{4}=\mathrm{R}_{5}=\mathrm{R}^{\prime}{ }_{1}=\mathrm{R}_{2}^{\prime}=\mathrm{R}^{\prime}{ }_{3}=\mathrm{R}_{4}^{\prime}=\mathrm{R}^{\prime}{ }_{5}=\mathrm{H} ; \mathrm{R}_{3}=\mathrm{F}$

4: $\mathrm{R}_{2}=\mathrm{R}_{3}=\mathrm{R}_{5}=\mathrm{R}_{1}^{\prime}=\mathrm{R}_{2}^{\prime}=\mathrm{R}^{\prime}{ }_{3}=\mathrm{R}^{\prime}{ }_{4}=\mathrm{R}^{\prime}{ }_{5}=\mathrm{H}_{3} \mathrm{R}_{2}=\mathrm{R}_{3}=\mathrm{F}$

5: $\mathrm{R}_{1}=\mathrm{R}_{3}=\mathrm{R}_{5}=\mathrm{R}^{\prime}{ }_{1}=\mathrm{R}_{2}{ }_{2}=\mathrm{R}^{\prime}{ }_{3}=\mathrm{R}_{4}^{\prime}=\mathrm{R}^{\prime}{ }_{5}=\mathrm{H} ; \mathrm{R}_{2}=\mathrm{R}_{4}=\mathrm{F}$

6: $\mathrm{R}_{3}=\mathrm{R}_{4}=\mathrm{R}_{5}=\mathrm{R}^{\prime}{ }_{1}=\mathrm{R}_{2}^{\prime}=\mathrm{R}^{\prime}{ }_{3}=\mathrm{R}_{4}^{\prime}=\mathrm{R}^{\prime}{ }_{5}=\mathrm{H} ; \mathrm{R}_{1}=\mathrm{R}_{2}=\mathrm{F}$

7: $\mathrm{R}_{2}=\mathrm{R}_{3}=\mathrm{R}_{5}=\mathrm{R}^{\prime}{ }_{1}=\mathrm{R}_{2}^{\prime}=\mathrm{R}^{\prime}{ }_{3}=\mathrm{R}^{\prime} 4=\mathrm{R}^{\prime}{ }_{5}=\mathrm{H} ; \mathrm{R}_{1}=\mathrm{R}_{4}=\mathrm{F}$

8: $\mathrm{R}_{2}=\mathrm{R}_{3}=\mathrm{R}_{4}=\mathrm{R}_{1}{ }_{1}=\mathrm{R}_{2}{ }_{2}=\mathrm{R}^{\prime}{ }_{3}=\mathrm{R}_{4}^{\prime}=\mathrm{R}^{\prime}{ }_{5}=\mathrm{H} ; \mathrm{R}_{1}=\mathrm{R}_{5}=\mathrm{F}$

9: $\mathrm{R}_{4}=\mathrm{R}_{5}=\mathrm{R}^{\prime}{ }_{1}=\mathrm{R}^{\prime}{ }_{2}=\mathrm{R}^{\prime}{ }_{3}=\mathrm{R}_{4}^{\prime}=\mathrm{R}^{\prime}{ }_{5}=\mathrm{H} ; \mathrm{R}_{1}=\mathrm{R}_{2}=\mathrm{R}_{3}=\mathrm{F}$

10: $\mathrm{R}_{3}=\mathrm{R}_{1}^{\prime}=\mathrm{R}_{2}^{\prime}=\mathrm{R}^{\prime}{ }_{3}=\mathrm{R}_{4}^{\prime}=\mathrm{R}_{5}^{\prime}=\mathrm{H} ; \mathrm{R}_{1}=\mathrm{R}_{2}=\mathrm{R}_{4}=\mathrm{R}_{5}=\mathrm{F}$

11: $\mathrm{R}_{1}=\mathrm{R}_{2}=\mathrm{R}_{3}=\mathrm{R}_{4}=\mathrm{R}_{5}=\mathrm{F} ; \mathrm{R}_{1}^{\prime}=\mathrm{R}_{2}{ }_{2}=\mathrm{R}^{\prime}{ }_{3}=\mathrm{R}_{4}^{\prime}=\mathrm{R}_{5}^{\prime}=\mathrm{H}$

12: $\mathrm{R}_{1}=\mathrm{R}_{2}=\mathrm{R}_{3}=\mathrm{R}_{4}=\mathrm{R}_{5}=\mathrm{H}_{3} \mathrm{R}_{1}{ }_{1}=\mathrm{R}_{2}^{\prime}=\mathrm{R}^{\prime}{ }_{3}=\mathrm{R}^{\prime}{ }_{4}=\mathrm{R}^{\prime}{ }_{5}=\mathrm{F}$

13: $\mathrm{R}_{1}=\mathrm{R}_{2}=\mathrm{R}_{3}=\mathrm{R}_{4}=\mathrm{R}_{5}=\mathrm{R}^{\prime}{ }_{1}=\mathrm{R}_{2}^{\prime}=\mathrm{R}^{\prime}{ }_{3}=\mathrm{R}_{4}^{\prime}=\mathrm{R}^{\prime}{ }_{5}=\mathrm{F}$ 


\section{PARTE EXPERIMENTAL}

Materiais. Acetofenona e benzaldeído foram adquiridos à Carlo Erba e usados como recebidos. 3-Fluorbenzaldeído; 4-fluorbenzaldeído; 3,4-difluorbenzaldeído; 3,5-difluorbenzaldeído; 2,3-difluorbenzaldeído; 2,5-difluorbenzaldeído; 2,6-difluorbenzaldeído; 2,3,4-trifluorbenzaldeído; 2,3,5,6-tetrafluorbenzaldeído; 2,3,4,5,6-pentafluorbenzaldeído e 2',3',4',5',6' -pentafluoracetofenona foram adquiridos à Aldrich Chemical e usados como recebidos.

Etanol e benzeno grau espectrofotométrico, hidróxidos de potássio e de sódio foram adquiridos ao Grupo Química. Benzeno foi seco sobre sódio metálico e destilado antes da sua utilização.

Sínteses. As chalconas 1-13, obtidas a partir de uma reação de condensação aldólica em meio básico ${ }^{20}$ entre acetofenona e os respectivos benzaldeídos (chalconas 1-11), entre 2', 3', 4', 5', 6'pentafluoracetofenona e benzaldeído (chalcona 12) e entre 2',3',4',5',6'-pentafluoracetofenona e 2,3,4,5,6-pentafluorbenzaldeído (chalcona 13) foram recristalizadas sucessivas vezes de EtOH/H $/ \mathrm{H}_{2}$. Os dados espectroscópicos listados a seguir estão de acordo com as estruturas propostas para 1-13.

chalcona (1) - RMN ${ }^{1} \mathrm{H} \delta$ (ppm): 8,20-7,40 (m, 10H aromáti$\cos ) ; 7,80\left(\mathrm{~d}, \mathrm{H}_{7}\right) ; 7,48\left(\mathrm{~d}, \mathrm{H}_{8}\right) ; \mathrm{RMN}{ }^{13} \mathrm{C} \delta(\mathrm{ppm}): 190,39(\mathrm{C}=\mathrm{O})$; $144,71\left(\mathrm{C}_{7}\right) ; 138,13\left(\mathrm{C}_{1}{ }^{\prime}\right) ; 134,79\left(\mathrm{C}_{1}\right) ; 132,67\left(\mathrm{C}_{4}{ }^{\prime}\right) ; 130,45\left(\mathrm{C}_{4}\right)$; $128,85\left(\mathrm{C}_{2}\right.$ e $\left.\mathrm{C}_{6}\right) ; 128,52\left(\mathrm{C}_{3}\right.$ e $\left.\mathrm{C}_{5}\right) ; 128,37\left(\mathrm{C}_{2}{ }^{\prime}=\mathrm{C}_{6}{ }^{\prime}\right.$ e $\left.\mathrm{C}_{3}{ }^{\prime}=\mathrm{C}_{5}{ }^{\prime}\right) ; 121,99$ $\left(\mathrm{C}_{8}\right) ; \mathrm{EM} \mathrm{m} / \mathrm{z}(\%): 208\left(\mathrm{M}^{+*}, 13\right) ; 207$ (80); 179 (18); 178 (18); 165 (9); 131 (36); 105 (31); 103 (38); 77 (100). IV v (cm-1) 1670,0 (C=O s-cis $) ; 1640,0(\mathrm{C}=\mathrm{O} s$-trans $)$; p.f. $=56-58{ }^{\circ} \mathrm{C}\left(\mathrm{lit}^{21}=57-58^{\circ} \mathrm{C}\right)$.

3-fluorchalcona (2) - RMN ${ }^{1} \mathrm{H} \delta$ (ppm): 7,99-7,10 (m, 9H, aromáticos); 7,74 (d, $\left.\mathrm{H}_{7}\right) ; 7,49\left(\mathrm{~d}, \mathrm{H}_{8}\right) . \mathrm{RMN}{ }^{13} \mathrm{C} \delta(\mathrm{ppm}):$ 189,65 (C=O); $162,70\left(\mathrm{~d}, \mathrm{C}_{3}\right) ; 142,87\left(\mathrm{C}_{7}\right) ; 137,59\left(\mathrm{C}_{1}{ }^{\prime}\right) ; 136,80\left(\mathrm{~d}, \mathrm{C}_{1}\right) ; 132,74$ $\left(\mathrm{C}_{4}{ }^{\prime}\right) ; 130,20\left(\mathrm{~d}, \mathrm{C}_{5}\right) ; 128,41\left(\mathrm{C}_{3}{ }^{\prime}=\mathrm{C}_{5}{ }^{\prime}\right) ; 128,27\left(\mathrm{C}_{2}{ }^{\prime}=\mathrm{C}_{6}{ }^{\prime}\right), 122,79$ $\left(\mathrm{C}_{8}\right) ; 115,60\left(\mathrm{~d}, \mathrm{C}_{2} \mathrm{e} \mathrm{C}_{6}\right) ; 114,40\left(\mathrm{~d}, \mathrm{C}_{4}\right) .\left(\mathrm{EM} \mathrm{m} / \mathrm{z}(\%): 226\left(\mathrm{M}^{+*}\right.\right.$, 55); 225 (44); 197 (18); 177 (7); 149 (27); 105 (50); 77 (100). IV v $\left(\mathrm{cm}^{-1}\right)$ 1671,0 $(\mathrm{C}=\mathrm{O} s$-cis $) ; 1654,0(\mathrm{C}=\mathrm{O}$ s-trans $)$. p.f. $=54-56{ }^{\circ} \mathrm{C}$.

4-fluorchalcona (3) - $\mathrm{RMN}{ }^{1} \mathrm{H} \delta$ (ppm): 7,99-7,10 (m, 9H, aromáticos); 7,76 (d, $\left.\mathrm{H}_{7}\right) ; 7,44\left(\mathrm{~d}, \mathrm{H}_{8}\right)$. $\mathrm{RMN}{ }^{13} \mathrm{C} \delta(\mathrm{ppm})$ : 189,81 (C=O); $115,80\left(\mathrm{~d}, \mathrm{C}_{3}\right) ; 143,13\left(\mathrm{C}_{7}\right) ; 137,77\left(\mathrm{C}_{1}{ }^{\prime}\right) ; 130,83\left(\mathrm{~d}, \mathrm{C}_{1}\right) ; 132,61$ $\left(\mathrm{C}_{4}{ }^{\prime}\right) ; 115,80\left(\mathrm{~d}, \mathrm{C}_{5}\right) ; 128,38\left(\mathrm{C}_{3}{ }^{\prime}=\mathrm{C}_{5}{ }^{\prime}\right) ; 128,22\left(\mathrm{C}_{2}{ }^{\prime}=\mathrm{C}_{6}{ }^{\prime}\right), 121,34$ $\left(\mathrm{C}_{8}\right) ; 130,10\left(\mathrm{~d}, \mathrm{C}_{2} \mathrm{e} \mathrm{C}_{6}\right) ; 163,70\left(\mathrm{~d}, \mathrm{C}_{4}\right)$. EM m/z (\%): $226\left(\mathrm{M}^{+\bullet}, 55\right)$; 225 (44); 197 (18); 177 (7); 149 (27); 105 (50); 77 (100). IV v $\left(\mathrm{cm}^{-1}\right)$ : $1671,8(\mathrm{C}=\mathrm{O}$ s-cis $) ; 1652,0(\mathrm{C}=\mathrm{O} s$-trans $)$. p.f. $=85-87^{\circ} \mathrm{C}$.

3,4-difluorchalcona (4) - $\mathrm{RMN}{ }^{1} \mathrm{H} \delta$ (ppm): 7,98-7,15 (m, 8H, aromáticos); 7,69 (d, $\left.\mathrm{H}_{7}\right) ; 7,45\left(\mathrm{~d}, \mathrm{H}_{8}\right) . \mathrm{RMN}^{13} \mathrm{C} \delta$ (ppm): 189,61 $(\mathrm{C}=\mathrm{O}) ; 151,60\left(\mathrm{dd}, \mathrm{C}_{3}\right) ; 150,60\left(\mathrm{dd}, \mathrm{C}_{4}\right) ; 142,06\left(\mathrm{C}_{7}\right) ; 137,64\left(\mathrm{C}_{1}{ }^{\prime}\right)$; $132,61\left(\mathrm{C}_{4}{ }^{\prime}\right) ; 131,98\left(\mathrm{C}_{1}\right) ; 128,54\left(\mathrm{C}_{3}{ }^{\prime}=\mathrm{C}_{5}{ }^{\prime}\right) ; 128,34\left(\mathrm{C}_{2}{ }^{\prime}=\mathrm{C}_{6}{ }^{\prime}\right) ; 122,61$ $\left(\mathrm{C}_{8}\right) ; 117,70\left(\mathrm{dd}, \mathrm{C}_{2}\right.$ e $\left.\mathrm{C}_{6}\right) ; 116,30\left(\mathrm{C}_{5}\right)$. (EM m/z (\%): $244\left(\mathrm{M}^{+*}\right.$, 100); 243 (35); 215 (14); 201 (5); 167 (31); 139 (18); 119 (6); 105 (33); 77 (38). IV $\vee\left(\mathrm{cm}^{-1}\right): 1670,6(\mathrm{C}=\mathrm{O} s-c i s) ; 1652,0(\mathrm{C}=\mathrm{O}$ $s$-trans $) ; 1606,0(\mathrm{C}=\mathrm{C} s$-cis $)$. p.f. $=110-111^{\circ} \mathrm{C}$.

3,5-difluorchalcona (5) - $\mathrm{RMN}{ }^{1} \mathrm{H} \delta(\mathrm{ppm}): 8,00-7,17(\mathrm{~m}, 8 \mathrm{H}$, aromáticos); 7,67 (d, $\left.\mathrm{H}_{7}\right) ; 7,49\left(\mathrm{~d}, \mathrm{H}_{8}\right) . \mathrm{RMN}{ }^{13} \mathrm{C} \delta(\mathrm{ppm}): 189,58$

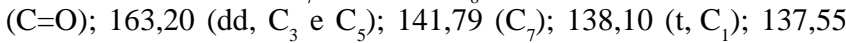
$\left(\mathrm{C}_{1}{ }^{\prime}\right) ; 133,12\left(\mathrm{C}_{4}{ }^{\prime}\right) ; 128,66\left(\mathrm{C}_{3}{ }^{\prime}=\mathrm{C}_{5}{ }_{5}\right) ; 128,47\left(\mathrm{C}_{2}{ }^{\prime}=\mathrm{C}_{6}{ }_{6}\right) ; 124,16\left(\mathrm{C}_{8}\right)$;

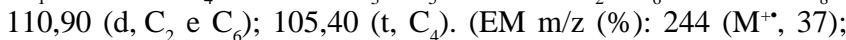
105 (53); 77 (100). IV $v\left(\mathrm{~cm}^{-1}\right): 1674,6(\mathrm{C}=\mathrm{O} s-c i s) ; 1650,0(\mathrm{C}=\mathrm{O} s$ trans $) ; 1614,0(\mathrm{C}=\mathrm{C} s$-cis $)$. p.f. $=107-109{ }^{\circ} \mathrm{C}$.

2,3-difluorchalcona $(\mathbf{6})-\mathrm{RMN}^{1} \mathrm{H} \delta(\mathrm{ppm}): 8,00-7,10(\mathrm{~m}, 8 \mathrm{H}$, aromáticos); 7,84 (d, $\left.\mathrm{H}_{7}\right) ; 7,64\left(\mathrm{~d}, \mathrm{H}_{8}\right)$. RMN ${ }^{13} \mathrm{C} \delta(\mathrm{ppm}): 189,77$ $(\mathrm{C}=\mathrm{O}) ; 137,43\left(\mathrm{C}_{1}{ }^{\prime}\right)$; 135,60 $\left(\mathrm{C}_{7}\right) ; 133,55\left(\mathrm{C}_{4}{ }^{\prime}\right)$; 128,49 $\left(\mathrm{C}_{2}{ }^{\prime}=\mathrm{C}_{6}{ }^{\prime}\right)$;

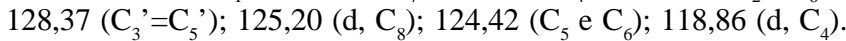
$\mathrm{EM} \mathrm{m/z} \mathrm{( \% ):} 244$ (M+*, 100); 243 (30); 225 (29); 215 (13); 214 (4); 201 (6); 196 (11); 195 (3); 167 (34); 139 (24); 119 (27); 105 (56);
77 (65). IV $\vee\left(\mathrm{cm}^{-1}\right): 1676,9(\mathrm{C}=\mathrm{O} s$-cis $) ; 1654,0(\mathrm{C}=\mathrm{O}$ s-trans $)$; $1610,0(\mathrm{C}=\mathrm{C} s$-cis $)$. p.f. $=69-71^{\circ} \mathrm{C}$.

2,5-difluorchalcona (7) - RMN ${ }^{1} \mathrm{H} \delta$ (ppm): 7,90-6,50 (m, 8H, aromáticos); 7,83 (d, $\left.\mathrm{H}_{7}\right) ; 7,59\left(\mathrm{~d}, \mathrm{H}_{8}\right) . \mathrm{RMN}{ }^{13} \mathrm{C} \delta$ (ppm): 198,88 $(\mathrm{C}=\mathrm{O}) ; 160,50\left(\mathrm{C}_{5}\right) ; 155,00\left(\mathrm{C}_{2}\right) ; 137,61\left(\mathrm{C}_{1}{ }^{\prime}\right) ; 135,90\left(\mathrm{C}_{7}\right) ; 133,06$ $\left(\mathrm{C}_{4}{ }^{\prime}\right) ; 128,64\left(\mathrm{C}_{3}{ }^{\prime}=\mathrm{C}_{5}{ }^{\prime}\right) ; 128,49\left(\mathrm{C}_{2}{ }^{\prime}=\mathrm{C}_{6}{ }^{\prime}\right) ; 125,30\left(\mathrm{C}_{8}\right) ; 118,40(\mathrm{~d}$, $\left.\mathrm{C}_{4}\right) ; 117,90\left(\mathrm{~d}, \mathrm{C}_{3}\right) ; 117,20$ (d, $\mathrm{C}_{6}$ ). EM m/z (\%): $244\left(\mathrm{M}^{+*}, 100\right) ; 243$ (29); 225 (36); 215 (18); 214 (5); 201 (7); 196 (11); 195 (6); 167 (42); 139 (29); 119 (36); 105 (67); 77 (82). IV $\vee\left(\mathrm{cm}^{-1}\right)$ : 1674,3 (C=O s-cis $) ; 1655,0(\mathrm{C}=\mathrm{O} s$-trans $) ; 1610,9(\mathrm{C}=\mathrm{C} s$-cis $)$. p.f. $=88-90{ }^{\circ} \mathrm{C}$.

2,6-difluorchalcona (8) - RMN ${ }^{1} \mathrm{H} \delta$ (ppm): 8,10-7,00 (m, 8H, aromáticos); 7,93 (d, $\left.\mathrm{H}_{7}\right) ; 7,84\left(\mathrm{~d}, \mathrm{H}_{8}\right) . \mathrm{RMN}{ }^{13} \mathrm{C} \delta$ (ppm): 189,85 $(\mathrm{C}=\mathrm{O}) ; 161,70\left(\mathrm{dd}, \mathrm{C}_{2}\right.$ e $\left.\mathrm{C}_{6}\right) ; 137,53\left(\mathrm{C}_{1}{ }^{\prime}\right) ; 132,83\left(\mathrm{C}_{4}{ }^{\prime}\right) ; 131,10(\mathrm{t}$, $\left.\mathrm{C}_{4}\right) ; 130,15\left(\mathrm{C}_{7}\right) ; 128,45\left(\mathrm{C}_{2}{ }^{\prime}=\mathrm{C}_{6}{ }_{6}\right) ; 128,34\left(\mathrm{C}_{3}{ }^{\prime}=\mathrm{C}_{5}{ }^{\prime}\right) ; 127,06\left(\mathrm{t}, \mathrm{C}_{8}\right)$; $111,70\left(\mathrm{C}_{3}\right.$ e C $\left._{5}\right)$. EM m/z (\%): $244\left(\mathrm{M}^{+\bullet}, 100\right) ; 243$ (20); 225 (34); 215 (13); 214 (9); 201 (5); 196 (11); 195 (3); 167 (44); 139 (27); 119 (33); 105 (56); 77 (78). IV $\vee\left(\mathrm{cm}^{-1}\right)$ : 1673,2 (C=O s-cis); 1650,0 $(\mathrm{C}=\mathrm{O} s$-trans $) ; 1615,5(\mathrm{C}=\mathrm{C} s$-cis $)$. p.f. $=58-60{ }^{\circ} \mathrm{C}$.

2,3,4-trifluorchalcona $(9)-\mathrm{RMN}^{1} \mathrm{H} \delta$ (ppm): 8,00-7,30 (m, 7H, aromáticos); 7,78 (d, $\left.\mathrm{H}_{7}\right) ; 7,60\left(\mathrm{~d}, \mathrm{H}_{8}\right) . \mathrm{RMN}^{13} \mathrm{C} \delta(\mathrm{ppm}): 189,37(\mathrm{C}=\mathrm{O})$; $150,40\left(\mathrm{dd}, \mathrm{C}_{2}\right) ; 151,90$ (dd, $\left.\mathrm{C}_{4}\right) ; 139,90$ (dt, $\left.\mathrm{C}_{3}\right) ; 137,36\left(\mathrm{C}_{1}{ }^{\prime}\right) ; 135,05$ $\left(\mathrm{C}_{7}\right) ; 132,98\left(\mathrm{C}_{4}{ }^{\prime}\right) ; 128,51\left(\mathrm{C}_{3}{ }^{\prime}=\mathrm{C}_{5}{ }^{\prime}\right) ; 128,31\left(\mathrm{C}_{2}{ }^{\prime}=\mathrm{C}_{6}{ }^{\prime}\right) ; 124,70\left(\mathrm{~d}, \mathrm{C}_{8}\right)$; 123,29 ( $\left.\mathrm{C}_{6}\right) ; 112,50\left(\mathrm{~d}, \mathrm{C}_{5}\right)$. EM m/z (\%): $262\left(\mathrm{M}^{+*}, 100\right) ; 261$ (15); 243 (21); 233 (11); 219 (6); 214 (9); 185 (30); 157 (29); 137 (29); 105 (67); 77 (78). IV $\vee\left(\mathrm{cm}^{-1}\right): 1674,6(\mathrm{C}=\mathrm{O} s$-cis $)$. p.f. $=78-80^{\circ} \mathrm{C}$.

2,3,5,6-tetrafluorchalcona (10) - RMN ${ }^{1} \mathrm{H} \delta$ (ppm): 8,00-7,09 (m, 6H, aromáticos); 7,89 $\left(\mathrm{d}, \mathrm{H}_{7}\right) ; 7,80\left(\mathrm{~d}, \mathrm{H}_{8}\right)$. RMN ${ }^{13} \mathrm{C} \delta(\mathrm{ppm})$ : $189,33(\mathrm{C}=\mathrm{O}) ; 137,21\left(\mathrm{C}_{1}{ }^{\prime}\right) ; 129,99\left(\mathrm{t}, \mathrm{C}_{8}\right) ; 128,00\left(\mathrm{C}_{2}{ }^{\prime}=\mathrm{C}_{6}{ }^{\prime}{ }^{\mathrm{e}}\right.$ $\left.\mathrm{C}_{3}{ }^{\prime}=\mathrm{C}_{5}{ }^{\prime}\right) ; 127,94\left(\mathrm{C}_{7}\right) ; 106,82\left(\mathrm{C}_{4}\right) . \mathrm{EM} \mathrm{m} / \mathrm{z}(\%): 280\left(\mathrm{M}^{+}, 40\right) ; 261$ (13); 232 (20); 203 (16); 175 (26); 155 (16); 127 (13); 105 (83); 77 (100). IV n $\left(\mathrm{cm}^{-1}\right): 1677,0(\mathrm{C}=\mathrm{O} s$-cis $) ; 1615,0(\mathrm{C}=\mathrm{C} s$-cis $)$. p.f. $=$ 119-121 ${ }^{\circ} \mathrm{C}$.

2,3,4,5,6-pentafluorchalcona (11) - $\mathrm{RMN}{ }^{1} \mathrm{H} \mathrm{d}$ (ppm): 8,01-7,50 (m, 5H, aromáticos); 7,83 $\left(\mathrm{d}, \mathrm{H}_{8}\right) ; 7,76\left(\mathrm{~d}, \mathrm{H}_{7}\right) . \mathrm{RMN}{ }^{13} \mathrm{C} \mathrm{d}(\mathrm{ppm})$ :

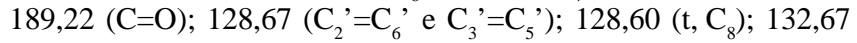
$\left(\mathrm{C}_{4}{ }^{\prime}\right) ; 127,66\left(\mathrm{C}_{7}\right) . \mathrm{EM} \mathrm{m} / \mathrm{z}(\%): 298\left(\mathrm{M}^{+}, 100\right) ; 279$ (12); 269 (5); 268 (1); 251 (11); 105 (97); 77 (167). IV n $\left(\mathrm{cm}^{-1}\right): 1670,0(\mathrm{C}=\mathrm{O} s-$ cis $) ; 1650,0(\mathrm{C}=\mathrm{O}$ s-trans $)$. p.f. $=147-148^{\circ} \mathrm{C}$.

2',3',4',5',6'-pentafluorchalcona (12) - $\mathrm{RMN}^{1} \mathrm{H} \mathrm{d}$ (ppm): 7,607,30 (m, 5H, aromáticos); 7,50 (d, $\left.\mathrm{H}_{7}\right) ; 7,01\left(\mathrm{~d}, \mathrm{H}_{8}\right) . \mathrm{RMN}{ }^{13} \mathrm{C} \mathrm{d}$ (ppm): 183,70 (C=O); 148,20 ( $\left.\mathrm{C}_{7}\right) ; 131,66\left(\mathrm{C}_{4}\right) ; 129,09\left(\mathrm{C}_{2}\right.$ e $\left.\mathrm{C}_{6}\right)$; 128,85 ( $\mathrm{C}_{3}$ e C $\left._{5}\right) ; 126,06\left(\mathrm{C}_{8}\right)$. EM m/z (\%): $298\left(\mathrm{M}^{+*}, 60\right) ; 297$ (100); 279 (2); 251 (3); 203 (16); 131 (14); 105 (83); 103 (24); 77 (22). IV $v\left(\mathrm{~cm}^{-1}\right): 1682,0(\mathrm{C}=\mathrm{O}$ s-cis $) ; 1665,0(\mathrm{C}=\mathrm{O}$ s-trans $) ; 1625,0(\mathrm{C}=\mathrm{C} s-$ cis $) ; 1650,0(\mathrm{C}=\mathrm{C} s$-trans $)$. p.f. $=90-94{ }^{\circ} \mathrm{C}$.

2,3,4,5,6,2',3',4',5',6'-decafluorchalcona (13) - RMN ${ }^{1} \mathrm{H} \delta$ (ppm): 7,60 (d, $\left.\mathrm{H}_{7}\right) ; 7,30\left(\mathrm{~d}, \mathrm{H}_{8}\right) . \mathrm{RMN}{ }^{13} \mathrm{C} \delta(\mathrm{ppm}): 182,86(\mathrm{C}=\mathrm{O})$; 131,97 ( $\left.\mathrm{C}_{7}\right) ; 130,07\left(\mathrm{C}_{8}\right)$. EM m/z (\%): $388\left(\mathrm{M}^{+*}, 40\right) ; 366$ (42); 341 (5). IV $\vee\left(\mathrm{cm}^{-1}\right): 1690,0(\mathrm{C}=\mathrm{O} s$-cis $) ; 1675,0(\mathrm{C}=\mathrm{O}$ s-trans $) ; 1625,0$ $(\mathrm{C}=\mathrm{C} s$-cis $) ; 1647,0(\mathrm{C}=\mathrm{C} s$-trans $)$.p.f. $=57-58{ }^{\circ} \mathrm{C}$.

Fotoquímica. Os cristais submetidos à irradiação foram preparados por evaporação na parede interna de um tubo de ensaio de Pyrex, a partir de uma solução cerca de $1,0 \mathrm{~mol} \mathrm{~L}^{-1} \mathrm{em} \mathrm{CH}_{2} \mathrm{Cl}_{2}$, e irradiados na região do ultravioleta $(\lambda=300 \mathrm{~nm})$ em atmosfera de argônio, por diferentes períodos de tempo. As irradiações foram efetuadas em um sistema composto de um carrossel e uma lâmpada Hanovia de vapor de mercúrio de média pressão $(450 \mathrm{~W})$, centrada em uma camisa de refrigeração de Pyrex equipada com circulação de água destilada.

A reatividade das chalconas estudadas está representada pelos resultados do consumo do isômero $\boldsymbol{E}$ das chalconas contra tempo de irradiação, como determinado por cromatografia em fase gasosa.

Métodos Gerais. Os pontos de fusão foram determinados num aparelho tipo Kofler e não foram corrigidos. 
Os espectros de massas foram obtidos através do acoplamento da cromatografia em fase gasosoa - espectrometria de massas computadorizada (CG-EM) em um espectrômetro de massas HewlettPackard (HP) modelo 5995, empregando uma coluna capilar HP-5, de sílica fundida, de $12 \mathrm{~m}$, adquirida à HP. Os espectros de massas foram registrados com o espectrômetro de massas operando a $70 \mathrm{eV}$.

Os espectros de $\mathrm{RMN}$ de ${ }^{1} \mathrm{H} \mathrm{e}{ }^{13} \mathrm{C}$ foram obtidos em um aparelho Bruker AC-200 ( ${ }^{1} \mathrm{H}: 200 \mathrm{MHz} ;{ }^{13} \mathrm{C}$ : 50,3 MHz), usando-se $\mathrm{CDCl}_{3}$ como solvente e TMS como padrão interno.

Os espectros na região do infravermelho (IV) foram obtidos em espectrofotômetro Perkin-Elmer modelo 1420, tendo sido utilizado filme líquido em $\mathrm{CCl}_{4}$, com cela de $\mathrm{NaCl}$ de espessura $0,1 \mathrm{~cm}$ e calibrado com filme de poliestireno a $1601 \mathrm{~cm}^{-1}$.

Os espectros na região do ultravioleta (UV) foram obtidos em espectrofotômetro Varian molelo DMS-80, tendo sido utilizada solução em $\mathrm{CH}_{2} \mathrm{Cl}_{2}$, com cela de quartzo de caminho ótico de $1,0 \mathrm{~cm}$.

Os cromatogramas em fase gasosa (CG) foram obtidos em um cromatógrafo a gás Varian modelo 3200 e empregando uma coluna capilar HP-5, de sílica fundida, de $15 \mathrm{~m}$, adquirida à HP. As condições empregadas na análise foram: $\mathrm{T}_{\text {detetor }}=300^{\circ} \mathrm{C} ; \mathrm{T}_{\text {injetor }}=280^{\circ} \mathrm{C} ; \mathrm{T}_{\text {coluna }}:$ inicial $=50$ ${ }^{\circ} \mathrm{C}, 1 \mathrm{~min}$; rampa de programação: $30^{\circ} \mathrm{C} / \mathrm{min}$; final $=280{ }^{\circ} \mathrm{C}, 15 \mathrm{~min}$.

\section{RESULTADOS}

A irradiação das chalconas $\mathbf{1}$ - 9 e $\mathbf{1 2}$ no estado sólido levou à formação de diferentes fotodímeros, com a estereoquímica dos ciclobutanos isoméricos obtidos sendo mostrada no Esquema 1.
A atribuição dos dímeros a suas respectivas estruturas baseou-se nos dados apresentados na literatura para a fotodimerização de alguns derivados de chalcona, nos padrões de multiplicidade dos sinais nos espectros de $\mathrm{RMN}{ }^{1} \mathrm{H}$, na interpretação dos deslocamentos químicos apresentados nos espectros de $\mathrm{RMN}{ }^{13} \mathrm{Ce}$, finalmente, pela comparação com os espectros de $\mathrm{RMN}{ }^{1} \mathrm{H}$ obtidos para alguns dos dímeros através de cálculos teóricos realizados por Montaudo e col. ${ }^{22}$ utilizando o programa LAOCOON II.

Dos quatro fotodímeros isoméricos formados a partir das $\boldsymbol{E}$ chalconas (Esquema 1), somente o isômero anti-cabeça-cauda ( $\varepsilon$ truxílico) deve-se apresentar como um sistema do tipo $\mathrm{A}_{2} \mathrm{~B}_{2}$ devido à presença, nesse derivado, de dois planos e um eixo $\mathrm{C}_{2}$ de simetria. No caso dos demais isômeros, devemos ter sistemas do tipo AA'BB', sendo que apenas o dímero sin-cabeça-cabeça apresenta um plano de simetria, enquanto os demais apresentam somente um eixo de simetria $\mathrm{C}_{2}$. Com relação ao padrão de multiplicidade dos sinais dos espectros de $\mathrm{RMN}{ }^{1} \mathrm{H}$ para os anéis ciclobutânicos, no sistema $\mathrm{A}_{2} \mathrm{~B}_{2}$ (E-truxílico) os sinais devem apresentar-se como sinais triplete, sendo que aqueles referentes ao sistema do tipo AA'BB' devem ser mais complexos e geralmente semelhantes a multipletos, com exceção do dímero sin-cabeça-cabeça, que deve apresentar-se como um mulipleto muito semelhante a um duplo-tripleto. A identificação dos diferentes dímeros obtidos neste trabalho foi baseada, portanto, nestas considerações e utilizando os dados de ressonância magnética nuclear de ${ }^{1} \mathrm{H}$ (Tabela 1) e ${ }^{13} \mathrm{C}$ (Tabela 2).

A irradiação de chalcona (1) e dos derivados apresentando apenas uma substituição por átomos de flúor, isto é $\mathbf{2}$ e $\mathbf{3}$, levou à forma-

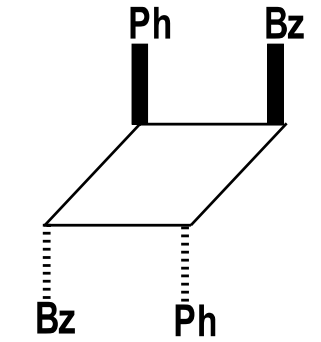

$\alpha$-truxílico sin-cabeça-cauda $(\sin -\mathrm{ht})$

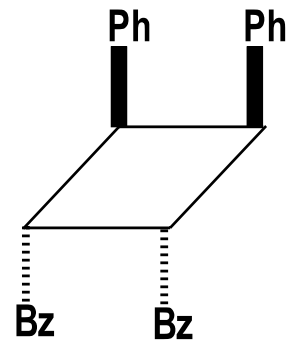

$\beta$-truxínico sin-cabeça-cabeça (sin-hh)

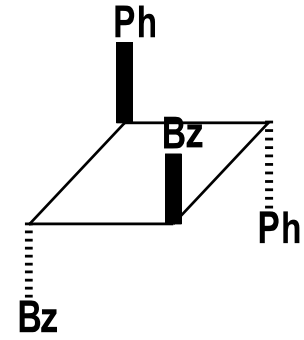

$\delta$-truxínico

anti-cabeça-cabeça (anti-hh)

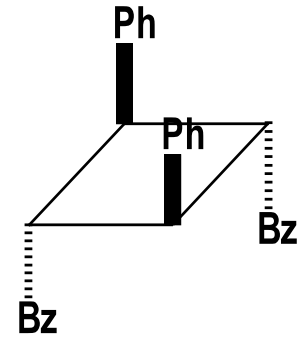

$\varepsilon$-truxílico

anti-cabeça-cauda (anti-ht)

Esquema 1

Tabela 1. Dados de RMN ${ }^{1} \mathrm{H}$ para os produtos obtidos na irradiação das chalconas $\mathbf{1}$ - $\mathbf{9}$ e $\mathbf{1 2}$ no estado sólido.

\begin{tabular}{|c|c|c|c|c|c|c|c|c|}
\hline \multirow[b]{5}{*}{ Chalcona } & \multicolumn{8}{|c|}{ Configuração } \\
\hline & \multicolumn{2}{|c|}{ anti-cabeça-cabeça } & \multicolumn{2}{|c|}{ sin-cabeça-cauda } & \multicolumn{2}{|c|}{ anti-cabeça-cauda } & \multicolumn{2}{|c|}{ sin-cabeça-cabeça } \\
\hline & \multicolumn{8}{|c|}{ Dímero } \\
\hline & \multicolumn{2}{|c|}{$\delta$-truxínico } & \multicolumn{2}{|c|}{$\alpha$-truxílico } & \multicolumn{2}{|c|}{ E-truxílico } & \multicolumn{2}{|c|}{$\beta$-truxínico } \\
\hline & \multicolumn{8}{|c|}{ Deslocamento Químico de ${ }^{1} \mathbf{H}$ (ppm) } \\
\hline 1 & 3,92 & 4,60 & 4,80 & 4,90 & 4,46 & 4,72 & & \\
\hline 2 & 3,88 & 4,50 & 4,74 & 4,83 & 4,42 & 4,68 & & \\
\hline 3 & 3,84 & 4,53 & 4,78 & 4,88 & 4,37 & 4,70 & & \\
\hline 4 & & & 4,74 & 4,82 & & & & \\
\hline 5 & & & 4,77 & 4,81 & & & & \\
\hline 6 & & & & & & & 4,87 & 5,22 \\
\hline 7 & & & & & & & 4,70 & 4,85 \\
\hline 8 & & & & & & & 4,85 & 5,33 \\
\hline 9 & & & & & & & 4,82 & 5,14 \\
\hline 12 & 3,80 & 4,19 & & & & & & \\
\hline
\end{tabular}


Tabela 2. Dados de $\mathrm{RMN}{ }^{13} \mathrm{C}$ para os produtos obtidos na irradiação das chalconas $\mathbf{1}$ - $\mathbf{9}$ e $\mathbf{1 2}$ no estado sólido.

\begin{tabular}{|c|c|c|c|c|c|c|c|c|}
\hline \multirow[b]{5}{*}{ Chalcona } & \multicolumn{8}{|c|}{ Configuração } \\
\hline & \multicolumn{2}{|c|}{ anti-cabeça-cabeça } & \multicolumn{2}{|c|}{ sin-cabeça-cauda } & \multicolumn{2}{|c|}{ anti-cabeça-cauda } & \multicolumn{2}{|c|}{ sin-cabeça-cabeça } \\
\hline & \multicolumn{8}{|c|}{ Dímero } \\
\hline & \multicolumn{2}{|c|}{$\delta$-truxínico } & \multicolumn{2}{|c|}{$\alpha$-truxílico } & \multicolumn{2}{|c|}{$\varepsilon$-truxílico } & \multicolumn{2}{|c|}{$\beta$-truxínico } \\
\hline & \multicolumn{8}{|c|}{ Deslocamento Químico de ${ }^{1} \mathrm{H}$ (ppm) } \\
\hline 1 & 47,58 & 47,86 & 42,37 & 50,59 & 44,70 & 49,07 & & \\
\hline 2 & 47,09 & 47,37 & 42,00 & 50,25 & 44,40 & 48,71 & & \\
\hline 3 & 47,40 & 47,64 & 41,45 & 51,00 & 44,00 & 49,00 & & \\
\hline 4 & & & 41,25 & 50,42 & & & & \\
\hline 5 & & & 41,95 & 50,02 & & & & \\
\hline 6 & & & & & & & 34,60 & 48,60 \\
\hline 7 & & & & & & & 39,26 & 47,37 \\
\hline 8 & & & & & & & 35,10 & 47,69 \\
\hline 9 & & & & & & & 34,20 & 48,47 \\
\hline 12 & 47,64 & 52,73 & & & & & & \\
\hline
\end{tabular}

ção de uma mistura de ciclobutanos, com predominância do derivado de configuração anti-cabeça-cabeça (dímero tipo ácido $\delta$ truxínico), além dos ciclobutanos sin-cabeça-cauda ( $\alpha$-truxílico) e anti-cabeça-cauda ( $\varepsilon$-truxílico). Na Figura 1 estão representados os sinais de $\mathrm{RMN}{ }^{1} \mathrm{H}$ obtidos experimentalmente para os hidrogênios $\mathrm{H}_{7}$ e $\mathrm{H}_{8}$ do produto de fotodimerização de 3-fluorchalcona (2). Nesse caso, é possível observar a presença de três conjuntos de sinais entre 3,8 e 5,0 ppm. O sinal de maior intensidade em 3,92 e 4,55 ppm foi atribuído ao dímero anti-cabeça-cabeça ( $\delta$-truxínico), em concordância com os valores obtidos em trabalhos anteriores para este tipo de dímero derivado de chalcona quando irradiada no estado fundido ${ }^{23}$

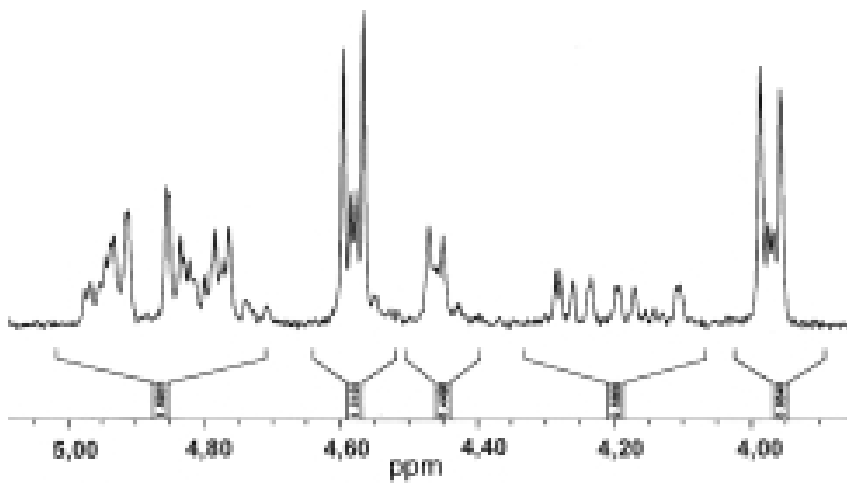

Figura 1. Espectro de $R M N^{1} H$ para o produto de irradiação no ultravioleta de 3-fluorchalcona (2) no estado sólido.

Os sinais multipletos absorvendo entre 4,8 e 5,0 ppm foram atribuídos aos dímeros sin-cabeça-cauda ( $\alpha$-truxílico) por comparação com os resultados de deslocamento químico apresentados por Toda e col. ${ }^{24}$ e obtidos experimentalmente pela irradiação de chalcona e alguns de seus derivados complexados com 1,1,6,6-tetrafenilexa-2,4diacetileno-1,6-diol. Finalmente, os sinais absorvendo na faixa entre 4,5 e 4,7 ppm foram atribuídos ao dímero anti-cabeça-cauda, não tendo sido encontrada na literatura nenhuma referência sobre a formação deste isômero no estudo de sistemas $\alpha, \beta$-insaturados. Espectros semelhantes foram encontrados para as chalconas $\mathbf{1}$ e $\mathbf{3}$.

Para 3-fluorchalcona (2) foi possível determinar-se, por integração dos sinais de $\mathrm{RMN}{ }^{1} \mathrm{H}$, a composição dos dímeros na mistura, tendo- se obtido as seguintes percentagens: anti-cabeça-cabeça ( $\delta$-truxínico), 48,6\%, sin-cabeça-cauda ( $\alpha$-truxílico), 30,0\% e anti-cabeça-cauda ( $\varepsilon$-truxílico), 21,4\%. Para as outras chalconas não foi possível ser estabelecida a composição da mistura de dímeros devido às baixas concentrações dos dímeros sin-cabeça-cauda e anti-cabeça-cauda presentes na mistura reacional.

Enquanto chalcona (1) teve a sua estrutura cristalina parcialmente destruída durante o processo de irradiação (fusão parcial da amostra), os derivados $\mathbf{2}$ e $\mathbf{3}$ mantiveram-se em seu estado cristalino.

A irradiação das chalconas $\mathbf{4}$ e $\mathbf{5}$ levou à formação exclusiva dos dímeros do tipo $\alpha$-truxílico com os sinais referentes aos hidrogênios $\mathrm{H}_{7}$ e $\mathrm{H}_{8}$ para estes ciclobutanos (multipleto na região de 4,7 a 4,9 ppm) (Figura 2) sendo coincidentes com aqueles apresentados para chalcona e alguns derivados que tiveram a sua estrutura determinada por cristalografia de raios- $\mathrm{X}^{24}$. Nas condições experimentais empregadas os compostos $\mathbf{4}$ e $\mathbf{5}$ levaram à produção de fotodímeros quase que quantitativamente, isto é, mais de $90 \%$ de rendimento, mesmo sob períodos prolongados de irradiação, não sendo evidenciada a formação de produtos secundários mesmo após mais de 10 horas de irradiação (Tabela 3).

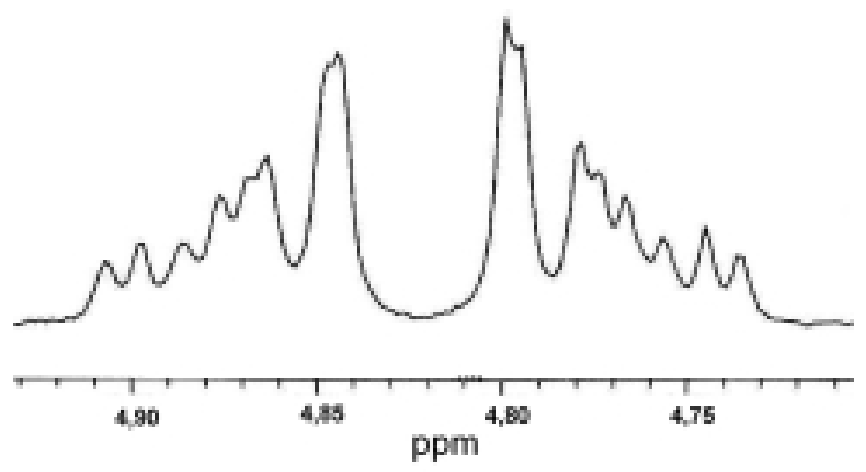

Figura 2. Espectro de $R M N^{l} H$ para o produto de irradiação no ultravioleta de 3,5-fluorchalcona (5) no estado sólido.

Os espectros de $\mathrm{RMN}^{1} \mathrm{H}$ para os derivados 6-9, na região de absorção do anel cliclobutano, estão mostrados na Figura 3. A atribuição desses sinais teve como base a análise da região dos deslocamentos químicos dos núcleos de hidrogênio, isto é $\mathrm{H}_{7}$ e $\mathrm{H}_{8}$, para os respectivos dímeros, bem como a sua multiplicidade. Na elucidação estrutural 
Tabela 3. Percentagem de fotodímeros formados na irradiação das chalconas 1-9 e 12, no estado sólido, como uma função do tempo de irradiação.

\begin{tabular}{cccc}
\hline Tempo (horas) & 1 & 3,5 & 10 \\
\hline \multicolumn{3}{c}{ Dímeros } \\
\hline Chalcona & $\%$ & $\%$ & $\%$ \\
\hline $\mathbf{1}$ & 0 & 16 & 32 \\
$\mathbf{2}$ & 3 & 17 & 59 \\
$\mathbf{3}$ & 2 & 40 & 78 \\
$\mathbf{4}$ & 40 & 95 & 100 \\
$\mathbf{5}$ & 32 & 94 & 100 \\
$\mathbf{6}$ & 2 & 37 & 66 \\
$\mathbf{7}$ & 0 & 2 & 3 \\
$\mathbf{8}$ & 0 & 38 & 61 \\
$\mathbf{9}$ & 48 & 94 & 100 \\
$\mathbf{1 2}$ & 0 & 8 & 15 \\
\hline
\end{tabular}

destes dímeros também foi considerada a região de deslocamento químico para os carbonos do anel ciclobutano, $\mathrm{C}_{7} \mathrm{e} \mathrm{C}_{8}$, conforme mostrado na Tabela 2. A confirmação destas estruturas foi feita por cromatografia de gás acoplada à espectrometria de massas. Os dados espectrométricos para estas chalconas mostraram que a formação do fotodímero sin-cabeça-cabeça ( $\beta$-truxínico) é feita de forma estereoespecífica. Para a chalcona mais substituída, isto é $\mathbf{9}$, observouse alto rendimento de fotocicloadição (mais de 90\%), mesmo após prolongados períodos de irradiação (mais do que 10 horas) (Tabela 3 ).

Para a fluorchalcona $\mathbf{1 2}$ foi verificada a existência de apenas um conjunto de sinais no espectro de $\mathrm{RMN}{ }^{1} \mathrm{H}$, o qual foi atribuído ao dímero de configuração tipo ácido $\delta$-truxínico por comparação com o espectro de chalcona (1). Apesar do espectro obtido indicar a possível formação dos outros dímeros, a baixa taxa de conversão para a sua formação impediu a confirmação da sua presença.

Os compostos apresentando altos graus de substituição por flúor, isto é, 10, 11 e 13 mostraram-se altamente reativos, com a possível formação de fotopolímeros e/ou produtos de decomposição, não tendo sido detectada a formação de ciclobutanos.

\section{DISCUSSÃO}

A reatividade fotoquímica no estado cristalino para as chalconas 1-13, da mesma forma que em solução ${ }^{25}$, é dependente tanto da posição quanto do número de átomos de flúor presentes no anel A e/ou no anel B da chalcona correspondente.

$\mathrm{O}$ estudo por ressonância magnética nuclear de ${ }^{1} \mathrm{H}$ (Tabela 1) e ${ }^{13} \mathrm{C}$ (Tabela 2) para o anel ciclobutano mostra que nas configurações sin-cabeça-cabeça e anti-cabeça-cauda os hidrogênios $\mathrm{H}_{7}$ e $\mathrm{H}_{8}$ e os carbonos $\mathrm{C}_{7}$ e $\mathrm{C}_{8}$ sofrem de efeitos de proteção e desproteção diferentes, tanto em modo quanto em intensidade, por parte das carbonilas e dos anéis aromáticos.

Nos resultados obtidos pela simulação em computador, utilizando o programa LAOCOON II, há dois conjuntos de multipletos os quais foram atribuídos por Montaudo ${ }^{22}$ como sendo pertencentes a sistemas típicos $\mathrm{A}_{2} \mathrm{~B}_{2}$ e AA'BB', respectivamente. O sinal apresentando um menor número de acoplamentos, e assemelhando-se muito a um tripleto, foi atribuído ao dímero anti-cabeça-cauda, enquanto que o sinal mais complexo, sugerindo um maior número de acoplamentos e, conseqüentemente, um sistema menos simétrico, pode ser atribuído ao dímero sin-cabeça-cabeça. Uma comparação entre os espectros apresentados na Figura 3 e os obtidos por simulação computacional por Montaudo e col. ${ }^{22}$ mostra que o ciclobutano resultante da irradiação das chalconas 6-9 é, possivelmente, de configuração sin-cabeça-cabeça (tipo ácido $\beta$-truxínico). Para este dímero, observa-se para $\mathrm{oH}_{7}$ um efeito de desproteção por anisotropia pela presença de um grupo carbonila no vértice oposto do anel ciclobutânico. Esse efeito deve ser muito efetivo devido a mudanças conformacionais sofridas pela molécula a fim de minimizar as interações desestabilizantes geradas pelas carbonilas que estão situadas em carbonos vicinais e em uma relação $\sin$. O mesmo não ocorre para a configuração anti-cabeça-cauda a qual, conseqüentemente, deve apresentar os sinais do núcleo $\mathrm{H}_{7}$ deslocados para campo mais alto (Tabela 1). Com relação ao hidrogênio $\mathrm{H}_{8}$, na configuracão sincabeça-cabeça esse núcleo sofre um efeito de desproteção por anisotropia muito efetivo exercido por um anel aromático situado no vértice oposto do anel ciclobutânico, o que não ocorre para $\mathrm{o}_{8}$ na configuração anti-cabeça-cauda. Conseqüentemente, este último também deve absorver em campo mais alto (Tabela 1).
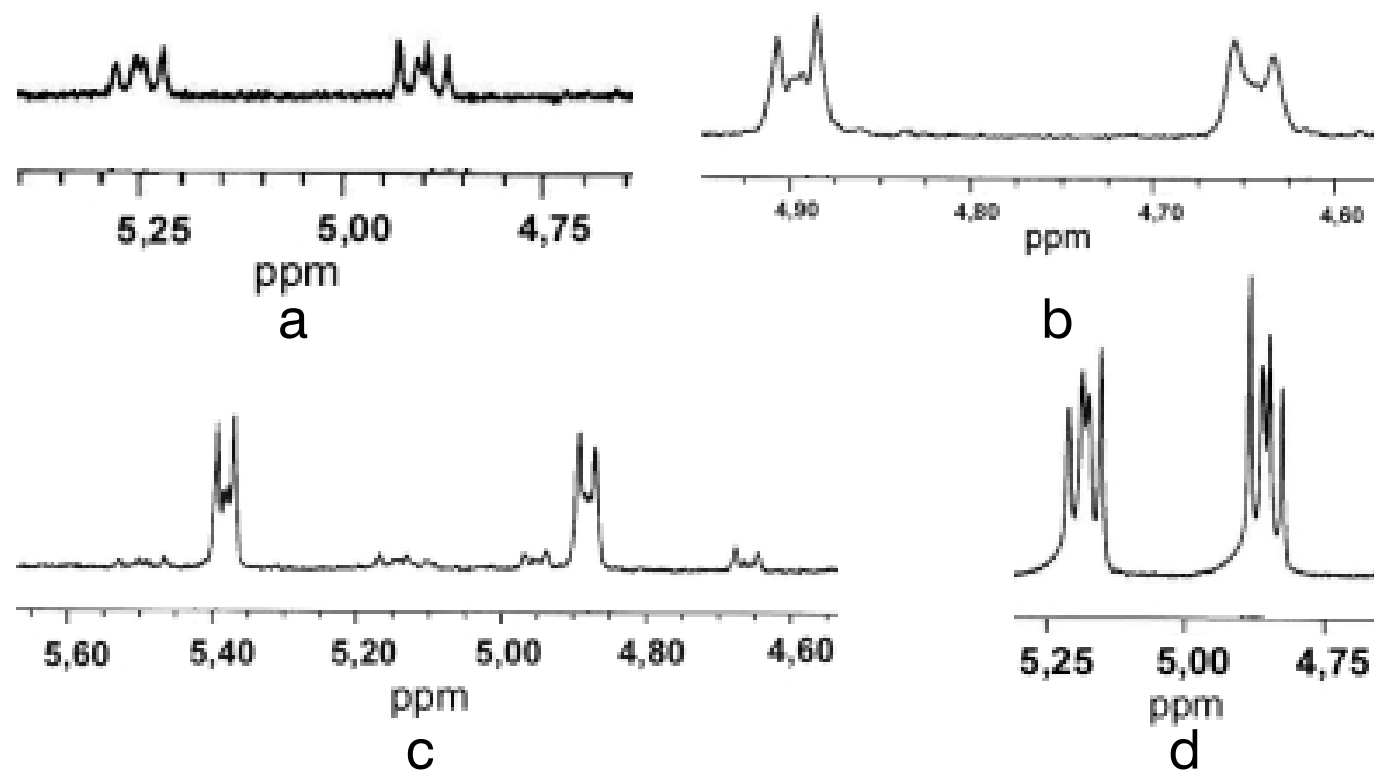

Figura 3. Espectro de $R M N^{1}$ H para o produto de irradiação no ultravioleta, no estado sólido, de: (a) 2,3-difluorchalcona (6); (b) 2,5-difluorchalcona (7); (c) 2,6-difluorchalcona (8); (d) 2,3,4-trifluorchalcona (9). 

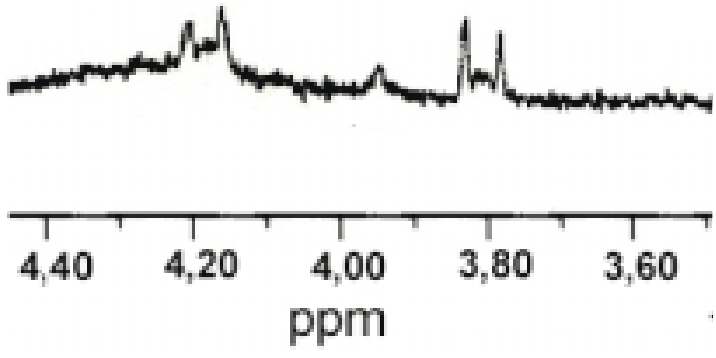

Figura 4. Espectro de $R M N^{1} \mathrm{H}$ para o produto de irradiação no ultravioleta de 2',3',4',5',6'-pentafluorchalcona (12) no estado sólido.

No caso dos núcleos de carbono (Tabela 2), esses centros estão dispostos de tal forma que o carbono $\mathrm{C}_{7}$ está ligado diretamente a um anel aromático enquanto $\mathrm{C}_{8}$ está ligado a um carbono carbonílico, justificando assim, em parte, um maior deslocamento químico para este último. No entanto, na configuração sin-cabeça-cabeça o núcleo $\mathrm{C}_{7}$ experimenta um efeito $\gamma$ de proteção pronunciado, gerado pela carbonila que se encontra situada no vértice oposto do anel ciclobutânico. $\mathrm{O}$ mesmo não acontece para $\mathrm{C}_{7}$ na configuração anticabeça-cauda e, conseqüentemente, este último deve absorver em campo mais baixo (Tabela 2). Para $\mathrm{C}_{8}$ temos que, tanto na configuração sin-cabeça-cabeça quanto na anti-cabeça-cauda, os efeitos $\gamma$ de proteção gerados pelos anéis aromáticos são praticamente semelhantes e, com isso, os carbonos $\mathrm{C}_{8}$ devem absorver praticamente na mesma região do espectro de RMN para ambos os fotodímeros (Tabela 2).

Os argumentos sugerindo que os núcleos de $\mathrm{H}$ e $\mathrm{C}$ nos espectros de RMN sofrem efeitos de proteção e desproteção mais pronunciados quando se encontram em uma relação eclipsada com uma carbonila ou um anel aromático situados em vértices opostos do anel ciclobutano estão baseados na comparação do deslocamento químico para os sinais apresentados pelos dímeros tipo $\alpha$-truxílico e $\delta$ truxínico (Tabelas 1 e 2). No caso do dímero tipo $\alpha$-truxílico, a absorção para os sinais referentes aos núcleos $\mathrm{H}_{7}, \mathrm{H}_{8}$ e $\mathrm{C}_{7}$ em campo mais baixo do que para o dímero tipo $\delta$-truxílico é consequiência, principalmente, dos efeitos de desproteção por anisotropia gerados pelas carbonilas e pelos anéis aromáticos, no caso dos hidrogênios, e de proteção $\gamma$ para os carbonos.

A única referência à formação do dímero de configuração do tipo sin-cabeça-cabeça ( $\beta$-truxínico) para um análogo de chalcona está relacionada ao produto da fotodimerização da dibenzalacetona, indicando para o deslocamento químico dos hidrogênios $\mathrm{H}_{7}$ e $\mathrm{H}_{8}$ valores de 5,5 e 5,9 ppm, respectivamente ${ }^{15}$. Esses valores de deslocamento químico encontrados em campo significativamente mais baixo para os dímeros sin-cabeça-cabeça possívelmente são conseqüência dos efeito de desproteção gerados pelos grupos substituintes presentes no anel ciclobutano quando na configuração sin.

A utilização da espectrometria de massas na confirmação da determinação da configuração cabeça-cabeça ou cabeça-cauda referente aos ciclobutanos é pouco conclusiva devido à facilidade de fragmentação do íon molecular com a perda de um grupo benzoíla (Ph-CO) e a ausência e/ou baixa intensidade dos sinais provenientes de fragmentações que seriam típicas para os ciclobutanos isoméricos. No entanto, a presença de sinais referentes à fragmentação resultante de uma clivagem homolítica do núcleo ciclobutânico, com a formação de um fragmento em baixa intensidade com $\mathrm{m} / \mathrm{z}=252$ para os derivados 6 e 8, sugere um dímero de configuração cabeça-cabeça. $O$ mesmo ocorre para os derivados 1-3, que formam dímeros de configuração cabeça-cabeça e, conseqüentemente, apresentam fragmen- tos de clivagem homolítica com $\mathrm{m} / \mathrm{z}=180$ e 252 , respectivamente. Um segundo fator que levou à proposição da formação de dímeros sin-cabeça-cabeça para os derivados contendo átomos de flúor na posição 2 é a presença de um sinal de baixa intensidade (<5\%) com massa $\mathrm{m} / \mathrm{z}=305$ nos espectros de massas dos compostos 6 e $\mathbf{8}$, e $\mathrm{m} / \mathrm{z}=341$ para 9 . Estes fragmentos são provenientes de um rearranjo tipo McLaferty, que somente é possível para o caso dos dímeros que adotem uma configuração $\sin ^{26}$.

De modo geral, os resultados apresentados neste trabalho mostram que a presença de átomos de flúor como substituintes nos anéis aromáticos influencia de forma marcante o comportamento fotoquímico das chalconas 1-13 quando irradiadas no estado cristalino. De acordo com os resultados apresentados na literatura, a baixa reatividade apresentada por chalcona (1) é conseqüência do seu arranjo cristalino, a qual pode apresentar-se em duas formas, tipo I e tipo II (p.f. $=59$ e $56^{\circ} \mathrm{C}$, respectivamente $)^{21,27}$, sendo que nos dois arranjos as distâncias entre as ligações duplas são maiores do que 4,3 Å. Assim, a ausência de reatividade está de acordo com o previsto pela teoria do controle topológico estabelecido por $\mathrm{Schmidt}^{8}$ que estabelece que compostos contendo ligações duplas separadas por mais de 4,2 A são fotoestáveis no estado sólido cristalino.

A obtenção de fotodímeros nos estudos realizados no presente trabalho no estado cristalino para a chalcona $\mathbf{1}$, contrariando os resultados apresentados na literatura, provavelmente se deve à forma pela qual se obteve os cristais que foram submetidos à irradiação. $\mathrm{O}$ processo de recristalização por evaporação sobre a superfície de um tubo de ensaio de Pyrex, feito neste trabalho, deve proporcionar a formação de um cristal com um grande número de falhas ou defeitos em sua estrutura cristalina, o que possivelmente deve favorecer a reação de fotodimerização. Isto também justificaria a sua liquefação em uma temperatura abaixo do seu ponto de fusão $\left(56 / 59{ }^{\circ} \mathrm{C}\right)$ durante o processo de irradiação, que foi realizado a $30^{\circ} \mathrm{C}$.

A engenharia cristalográfica proposta por $\mathrm{Schmidt}^{8}$ estabelece que a presença de átomos de halogênio como cloro, principalmente em compostos diclorossubstituídos, aumenta drasticamente o rendimento de formação de ciclobutanos e direciona o processo de fotodimerização dos derivados de ésteres cinâmicos para a obtenção de ciclobutanos do tipo $\beta$-truxínico. Nossos estudos permitiram verificar que a presença de apenas um átomo de flúor como substituinte sobre o resíduo cinâmico de chalconas (anel A), independentemente de sua posição, isto é 3-fluorchalcona (2) ou 4-fluorchalcona (3), não é suficiente para provocar algum tipo de mudança estrutural no arranjo cristalino, quando comparados com a chalcona, como pode ser verificado pela distribuição dos produtos formados (Tabelas 1 e 2), mas acarreta um aumento significativo em sua reatividade (Tabela 3 ).

Com relação às chalconas contendo dois ou três átomos de flúor substituídos no anel A (resíduo cinamoíla), a sua fotorreatividade é significativamente alterada, sendo altamente dependente da posição dos átomos de flúor sobre o anel. Uma comparação entre 4 e 5 mostra que, em ambos os casos, há uma reação estereosseletiva com a formação exclusiva do dímero sin-cabeça-cauda ( $\alpha$-truxílico), o que possivelmente reflete uma forma de empacotamento preferencial, que se deve a possíveis intrerações intermoleculares durante o processo de cristalização por evaporação. Os maiores rendimentos observados para os derivados $\mathbf{4}$ e $\mathbf{5}$ quando comparados com o obtido para chalcona (1) (Tabela 3 ) podem estar relacionados com a aproximação das ligações duplas reativas como conseqüência da substituição sobre o anel aromático cinamoílico.

As chalconas 6-9, todas substituídas por flúor na posição 2, apresentam como característica a preferência para a fotodimerização estereosseletiva com formação de dímeros sin-cabeça-cabeça. Novamente, uma explicação para esse comportamento pode estar relacionada com as interações (intermoleculares) provocadas pelos 
substituintes durante o processo de recristalização, o que altera a forma de empacotamento no estado cristalino. A diferença de comportamento entre os compostos 4 e 5 e 6-9, que apresentam flúor na posição 2, isto é a formação de fotodímeros de configuração sincabeça-cauda para os primeiros e sin-cabeça-cabeça para os últimos, deve estar relacionada exclusivamente à presença do flúor na posição 2. Durante o processo de recristalização por evaporação, as moléculas tornam-se cada vez mais próximas umas das outras, de tal forma que, no arranjo topoquímico mais favorável, as interações repulsivas tenderão a ser minimizadas enquanto que as atrativas tenderão a um máximo. A presença do átomo de flúor na posição 2 faz com que essas moléculas adotem, em solução, uma conformação sin entre o átomo de flúor e a ligação olefínica ${ }^{28}$. É possível, então, que essa preferência conformacional torne o empacotamento das moléculas no estado cristalino que leva à produção dos ciclobutanos sincabeça-cabeça, termodinamicamente mais estável do que aquela levando ao isômero sin-cabeça-cauda. No primeiro caso pode-se postular a presença de possíveis interações atrativas intermoleculares entre os átomos de flúor na posição 2 , sendo que na configuração sin-cabeça-cauda essas interações atrativas não existiriam. $\mathrm{O}$ mesmo efeito não deve estar presente no empacotamento sin-cabeça-cabeça para os derivados 4 e 5 e, conseqüentemente, estes derivados preferem o arranjo que leva à formação do ciclobutano sin-cabeça-cauda.

$\mathrm{O}$ alto rendimento do derivado 9 quando comparado a 6-8 possivelmente reflete uma maior interação intermolecular estabilizante, gerada pelo maior número de átomos de flúor, conseqüentemente diminuindo as distâncias entre as ligações duplas. Alternativamente, uma explicação para os altos rendimentos encontrados no processo de dimerização dos compostos 4, 5 e 9 pode estar relacionada ao fato de que provavelmente estes compostos apresentem uma estrutura cristalina para os reagentes muito semelhante tanto à do estado de transição quanto à do produto de fotodimerização, sendo que, nesse caso, a reação se processaria via um mecanismo cristal a cristal, justificando assim a sua alta estereosseletividade.

A ausência de mudanças na forma cristalina e a menor reatividade fotoquímica apresentadas por 12 (Tabela 3), quando comparado aos demais compostos fluorados e à chalcona, é conseqüência do efeito gerado pelos substituintes presentes no anel benzoílico sobre a sua estrutura cristalina. Esse efeito, diferente das demais chalconas estudadas, pode ser justificado por uma mudança no seu equilíbrio conformacional, uma vez que $\mathbf{1 2}$ apresenta uma maior percentagem do confôrmero s-trans em solução quando comparado a $\mathbf{1}$. Isto é conseqüência da menor coplanaridade do grupo benzoílico $\mathrm{Ar}-\mathrm{C}=\mathrm{O}$ com o grupo estirila restante da molécula, ou seja $\mathrm{CH}=\mathrm{CH}-\mathrm{Ph}^{29}$. Esse arranjo de menor coplanaridade pode estar dificultando o empacotamento molecular necessário à reação de fotodimerização no estado cristalino de $\mathbf{1 2}$ e, conseqüentemente, acarretando uma diminuição em sua reatividade.

Contrariamente a 12, a alta instabilidade fotoquímica apresentada pelos compostos 10, 11 e 13, levando a produtos de polimerização e/ou decomposição, com a destruição completa de sua forma cristalina, possivelmente reflete a alta reatividade apresentada por compostos polifluorados, como demonstrado para a decafluorbenzofenona ${ }^{30,31}$.

\section{CONCLUSÃO}

Em conclusão, este trabalho demonstra inequivocamnete que a fotoquímica de chalcona e de seus derivados fluorados, no estado sólido, depende não só do número de átomos de flúor substituídos nos anéis A e/ou B mas também da sua posição. Assim, a irradiação de 3- e 4-fluorchalcona resulta em uma distribuição de fotodímeros semelhante à de chalcona, apesar de serem mais reativas, sendo que nestes três casos a formação de produtos é consequiência da estrutura do cristal que deve apresentar um grande número de falhas ou defeitos. Por outro lado, a fotólise de chalconas di- ou trifluoradas leva à formação estereosseletiva de fotodímeros, o que demonstra a presença de uma forma de empacotamento preferencial devida a possíveis intrerações intermoleculares durante o processo de cristalização por evaporação. O comportamento fotoquímico de pentafluorchalcona substituída no anel B é dependente da sua conformação, com o arranjo de menor coplanaridade entre os cromóforos benzoíla e estirila daí resultante sendo responsável pela menor reatividade desta chalcona. Finalmente, chalconas tetra- e pentafluoradas no anel A são extremamente reativas, não levando, entretanto, a produtos de fotodimerização.

\section{AGRADECIMENTOS}

Este trabalho foi financiado pelo Conselho Nacional de Desenvolvimento Científico e Tecnológico (CNPq), Coordenação de Aperfeiçoamento de Pessoal do Ensino Superior (CAPES) e Fundação de Amparo à Pesquisa do Estado do Rio de Janeiro (FAPERJ). JCNF gostaria de agradecer ao CNPq a concessão de Bolsa de Produtividade em Pesquisa.

\section{REFERÊNCIAS}

1. Ramamurthy, V.; Venkatesan, K.; Chem. Rev. 1987, 87, 433.

2. Ramamurthy, V.; Venkatesan, K., Photochemistry in Organized and Constrained Media Ramamurthy, V. (ed.), VCH Publishers, 1991, NY.

3. Libermann, C.; Chem. Ber. 1889, 22, 124.

4. Libermann, C.; Chem. Ber. 1889, 22, 782.

5. Schmidt, G. M. J.; J. Chem. Soc. 1964, 2014.

6. Cohen, M. D.; Schmidt, G. M. J.; J. Chem. Soc. 1964, 1996.

7. Cohen, M. D.; Schmidt, G. M. J.; Sonntag, F. I.; J. Chem. Soc. 1964, 2000.

8. Schmidt, G. M. J.; Pure Appl. Chem. 1971, 27, 647.

9. Craig, D. P.; Sarti-Fantoni, P.; J. Chem. Soc., Chem. Commun. 1966, 742.

10. a) Bart, J. C. J.; Schmidt, G. M. J.; Isr. J. Chem. 1971, 9, 429; b) Heller, E.; Schmidt, G. M. J.; Isr. J. Chem. 1971, 9, 449.

11. Chandross, E. A.; Ferguson, J.; J. Chem. Phys. 1966, 45, 3564.

12. Cohen, M. D.; Angew. Chem. Int. Ed. Engl. 1975, 14, 386.

13. Garcia-Garibay, M. A.; Constable, A. E.; Jernelius, J.; Choi, T.; Cizmeciyan, D.; Shin, S. H., L. Echegoyen and A. E. Kaifer (eds). Physical Supramolecular Chemistry, 1996, p. 289.

14. Craig, D. P.; Lindsay, R. N.; Mallet, C. P.; Chem. Phys. 1984, 89, 187.

15. Green, B. S.; Schmidt, G. M. J.; Tetrahedron Lett. 1970, 4253.

16. Gnanaguru, K.; Ramasubbu, N.; Venkatesan, K.; Ramamurthy, V.; J. Org. Chem. 1985, 50, 2337.

17. Rabinovich, D. R.; Schmidt, G. M. J.; J. Chem. Soc. (B), 1970, 6.

18. Dihurjati, M. S. K.; Sarma, J. A. R. P.; Desiraju, G. R.; J. Chem. Soc., Chem. Commun. 1991, 1702.

19. Venugopalan, P.; Rao, T. B.; Vengatesan, K.; J. Chem. Soc., Perkin Trans. 2 1991, 981 .

20. Kohler, E. P.; Chadwell, H. M.; Org. Synth. Coll.; John Wiley: New York, 1932, Vol I, p. 78.

21. Rabinovich, D.; J. Chem. Soc. (B) 1970, 11.

22. Montaudo, G.; Caccamese, S.; Librando, V.; Org. Mag. Res. 1974, 6, 534

23. Caccamese, S.; McMillan, J. A.; Montaudo, G., J. Org. Chem. 1978, 43, 2703.

24. Toda, F.; Tanaka, K.; J. Chem. Soc., Chem. Commun. 1983, 593.

25. Cesarin-Sobrinho, D.; Netto-Ferreira, J. C., a ser publicado.

26. Stewart, D.; Robertson, G. W.; Morrison I. M.; Rap. Comm. Mass Spect. 1992, 6,45 .

27. Ohkura, K.; Kashino, S; Haisa, M.; Bull. Chem. Soc. Jpn. 1973, 49, 627.

28. Abraham, R. J.; Angioloni, S.; Edgar, M.; Sancassan, F.; J. Chem. Soc., Perkin Trans. 2 1997, 41.

29. Cesarin-Sobrinho, D.; Braz-Filho, R.; Netto-Ferreira, J. C.; Quim. Nova 2001, 24, 604

30. Dedinas, J.; Regan, T. H.; J. Phys. Chem. 1972, 76, 3926.

31. Boate, D. R.; Johnston, L. J; Scaiano, J. C.; Can. J. Chem. 1989, 67, 927. 